\title{
Are solution processed CsPbl3 perovskite nanocrystalline films similar to traditional crystalline semiconductors?
}

\section{Kanishka Kobbekaduwa}

Clemson University https://orcid.org/0000-0002-3414-741X

Exian Liu

Clemson University https://orcid.org/0000-0002-1031-7891

\section{Qian Zhao}

National Renewable Energy Laboratory

\section{Pan Adhikari}

Clemson University https://orcid.org/0000-0002-2515-6314

\section{Chendi Xie}

Clemson University

\section{Jianbing Zhang}

Huazhong University of Science and Technology https://orcid.org/0000-0003-0642-3939

\section{Ying Shi}

Jilin University

\section{Haimei Zheng}

Lawrence Berkeley National Laboratory https://orcid.org/0000-0003-3813-4170

\section{Dawen Li}

The University of Alabama

\section{Hugo Sanabria}

Clemson University https://orcid.org/0000-0001-7068-6827

\section{Ming Hu}

University of South Carolina

\section{Tong Cai}

Brown University https://orcid.org/0000-0002-4468-6767

\section{Ou Chen}

Brown University https://orcid.org/0000-0003-0551-090X

\section{Rahul Rao}

Air Force Research Laboratory https://orcid.org/0000-0002-6415-0185

\section{Apparao Rao}

Clemson University https://orcid.org/0000-0002-1450-3499

\section{Matthew Beard}


National Renewable Energy Laboratory

\section{Joseph Luther}

National Renewable Energy Laboratory https://orcid.org/0000-0002-4054-8244

Jianbo Gao ( $\sim$ jianbogao.nano@gmail.com )

Clemson University https://orcid.org/0000-0001-6203-5338

\section{Article}

Keywords: ultrafast photocurrent spectroscopy, perovskite materials, CsPbl3

Posted Date: September 29th, 2021

DOl: https://doi.org/10.21203/rs.3.rs-912920/v1

License: (c) (i) This work is licensed under a Creative Commons Attribution 4.0 International License. Read Full License 


\section{Are solution-processed $\mathrm{CsPbI}_{3}$ perovskite nanocrystal films similar to traditional semiconductors?}

Kanishka Kobbekaduwa ${ }^{1} \uparrow$, Exian Liu ${ }^{1} \uparrow$, Qian Zhao ${ }^{2} \uparrow$, Pan Adhikari ${ }^{1}$, Chendi Xie ${ }^{1}$, Jianbing Zhang ${ }^{3}$, Ying Shi ${ }^{4}$, Haimei Zheng ${ }^{5}$, Dawen $\mathrm{Li}^{6}$, Hugo Sanabria ${ }^{1}$, Ming Hu${ }^{7}$, Tong $\mathrm{Cai}^{8}, \mathrm{Ou}$ Chen $^{8}$, Rahul Rao ${ }^{9}$, Apparao M. Rao ${ }^{1}$, Matthew C. Beard ${ }^{2}$, Joseph M. Luther ${ }^{2}$, Jianbo Gao $^{1 *}$

${ }^{1}$ Department of Physics and Astronomy, Ultrafast Photophysics of Quantum Devices Laboratory, Clemson University, Clemson, SC, 29634, United States

${ }^{2}$ Chemistry \& Nanoscience Center, National Renewable Energy Laboratory, Golden, CO, 80401, United States

${ }^{3}$ School of Optical and Electronic Information, Huazhong University of Science and Technology, Wuhan 430074, P. R. China

${ }^{4}$ Institute of Atomic and Molecular Physics, Jilin Provincial Key Laboratory of Applied Atomic and Molecular Spectroscopy, Jilin University, Changchun, 130012, P. R. China

${ }^{5}$ Materials Sciences Division, Lawrence Berkeley National Laboratory, Berkeley, CA, 94720, United States

${ }^{6}$ Department of Electrical and Computer Engineering, Center for Materials for Information Technology, The University of Alabama, Tuscaloosa, AL, 35487, United States

${ }^{7}$ Department of Mechanical Engineering, University of South Carolina, Columbia, SC, 29208, United States

${ }^{8}$ Department of Chemistry, Brown University, Providence, RI, 02912, United States

${ }^{9}$ Materials and Manufacturing Directorate, Air Force Research Laboratory, Wright-Patterson Air Force Base, Dayton, OH, 45433, United States

$\dagger$ These authors contributed equally to this work.

*Corresponding author. Email: jianbogao.nano@gmail.com

Abstract: We use ultrafast photocurrent spectroscopy with a sub-25 picosecond resolution to address a long-standing question in emergent perovskite materials: are solution-processed $\mathrm{CsPbI}_{3}$ perovskites nanocrystal films similar to their counterparts semiconductors including GaAs and Si? Prior to carrier trapping, all semiconductors show similar transition of transport mechanism from the band-like transport due to the longitudinal optical (LO) phonon scattering in the higher temperature region to a combination of band-like and hopping transport mechanisms in the lower temperature region. The critical difference is that $\mathrm{CsPbI}_{3}$ demonstrate the strongest carrier-LO phonon interaction among all semiconductors, most likely due to the unique cation-halogen bond arrangements within the highly symmetric lattice structure. This ultrafast dynamics work establish a foundation of fundamental carrier transport understating for perovskite optoelectronics. 


\section{Introduction}

Despite exhibiting superior device performance in photonics, electronics, and optoelectronics, it remains unclear why emergent perovskite semiconductors, which are typically solution-processed at low temperatures, are similar in nature to those of benchmark semiconductors such as GaAs or $\mathrm{Si}$. In particular, the fundamental understanding of how the electrical transport properties of nanoand micro-crystalline perovskites differ from those of bulk crystalline semiconductors is still unclear. For example, perovskite solar cells have achieved more than $25 \%$ power conversion efficiency (1), while the efficiency of the current dominant silicon photovoltaic technology is $\sim 22$ $\%$. Perovskite nanocrystal X-ray detectors acquire the same imagery with more than two orders of magnitude lower radiation dosage than the traditional silicon-based photodiode(2). Perovskite nanocrystal light emitting diodes (LEDs) generate purer colors than their III-V and alloy-dominant semiconductor counterparts due to narrowband emission in the range of $<50 \mathrm{~nm}(3)$. Such insights into their transport properties will therefore enable the engineering of perovskite-based devices with superior device performance and efficiency. Furthermore, these insights could expedite the predictive discovery of future-generation technologies beyond current perovskite materials.

Typical solution synthesized and processed perovskites have an $\mathrm{ABX}_{3}$ chemical composition, wherein A=Methylammonium (MA), Formamidinium (FA), $\mathrm{Cs}$, etc.; $\mathrm{B}=\mathrm{Pb}, \mathrm{Sn}$, etc.; and $\mathrm{X}=\mathrm{Cl}$, $\mathrm{Br}$, I. Due to their low-temperature synthesis and processing, perovskites have the advantage of low-cost processing and flexibility on substrates but suffer from high defect densities. Common defects include grain boundaries and dislocations, while shallow point defects of both acceptor and donor type vacancies have also been reported $(4,5)$. In addition to reducing device performance and stability, these defects often overshadow our understanding of the carrier transport property, and most transport studies are dominated by carrier hopping transport in the energetic landscape of defect states. Typical trapping events occur in a wide temporal range from $\sim$ picoseconds (ps) to microseconds $(\mu \mathrm{s})$, as demonstrated in our previous work $(\sigma)$. Because of their ultrafast time resolution range from sub-ps to 10s of ps, time-resolved optical laser spectroscopies seem be able to unravel pre-trapping carrier dynamics. Two approaches categorize these spectroscopies. The first is based on the photon emission mechanism, which includes time-resolved photoluminescence (TRPL) and the streak camera technique(7). The second is the pump-probe mechanism, which includes transient absorption(8), THz-time domain spectroscopy (THz-TDS)(9-11), and other pump-probe approaches $(12,13)$. As schematically indicated in Fig. 1a, in the absence of an electrical field to 'push' the carriers, they spatially diffuse in a short-range domain confined to a couple of nanometers(14). As a result, these photon-in and photon-out techniques characterize carrier diffusion dynamics, in spite of that they have uncovered many novel ultrafast phenomena such as slow hot carrier cooling(8), efficient many-body interactions(15), and large polaron effects(16). However, for solar cells, LEDs, and photodetectors, the carrier drift dynamics (parameters including mobility, lifetime, and drift length, etc.) is the backbone of transport mechanism, in particular, when devices are at work, i.e., in operando conditions.

The electrical field in operando devices exerts a force or 'push' the carriers, enabling an understanding of carrier drift dynamics, in particular, in a long-range transport. For example, in a photoconductive device, the time-resolved photoconductivity techniques such as the time-of-flight photocurrent(17) and photo-CELIV (charge extraction by linearly increasing voltage) characterize the carrier drift properties including carrier mobility, lifetime, and drift length(18) (Fig. 1b). However, their time resolution is rather slow $(\sim 10 \mathrm{~s} n s)(19)$ and is much longer than the trapping events in most solution-processed perovskites. Thus, most measurements are influenced by defect 
states. Although ultrafast THz-TDS can derive carrier properties including mobility and lifetime at the same time based on the classic Drude model, the carriers are still confined to nanoscale domain, as demonstrated in the early studies with CdSe nanocrystals as well as perovskites(10, 20). In the end, there are scarce studies that shed light on the drift dynamics in devices before carrier trapping, as well as long-range transport features.

a

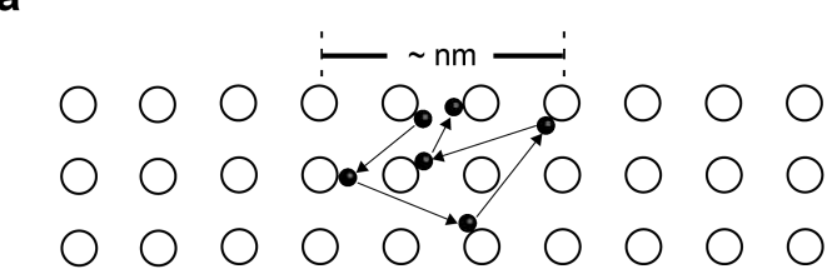

Diffusion transport

Photoluminescence measurements 1. time-resolved photoluminescence

2. streak camera technique

Pump-probe measurements

1. transient absorption

2. terahertz time domain spectroscopy

b
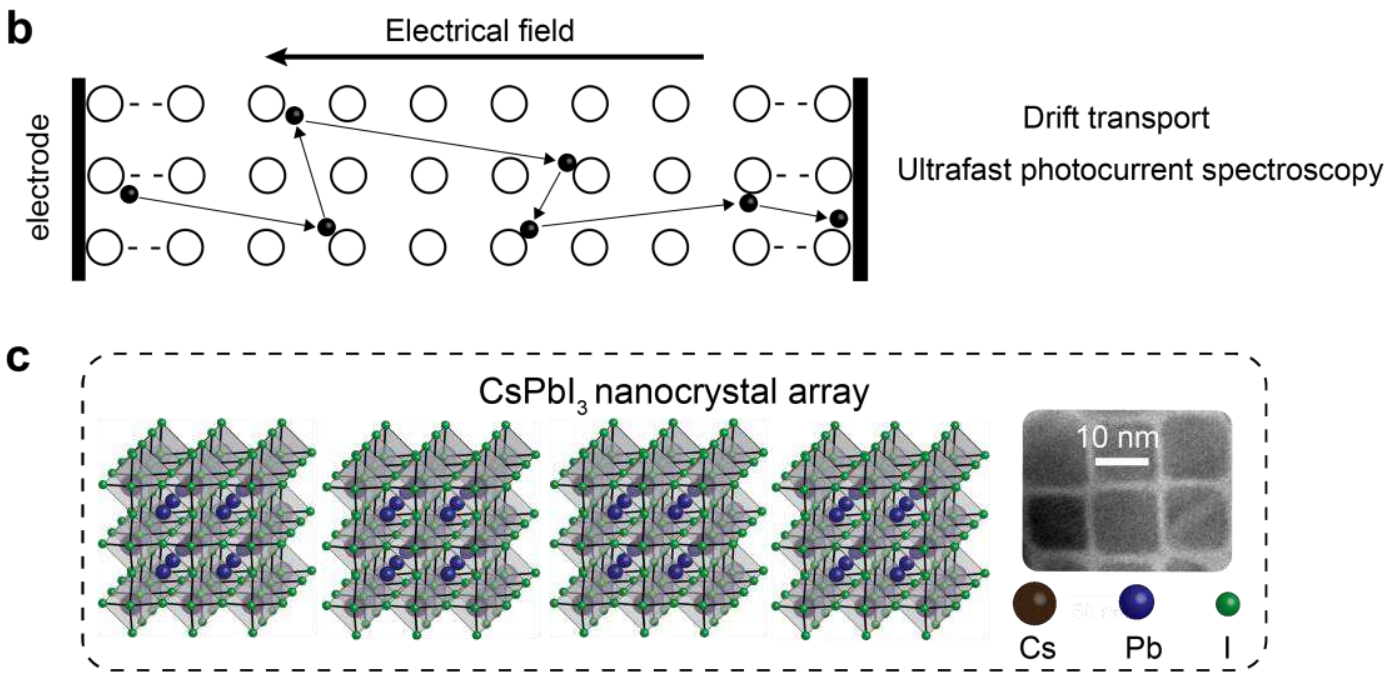

d

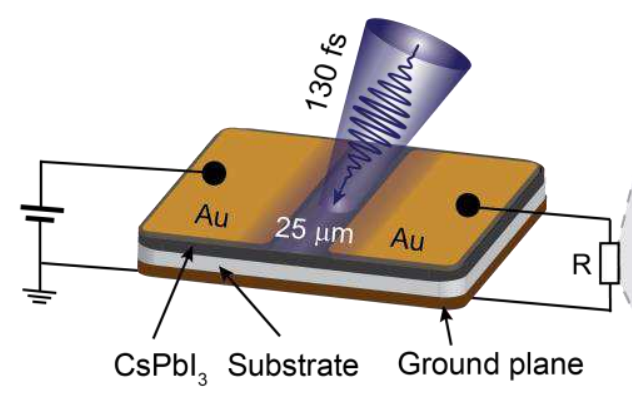

Sampling oscilloscope

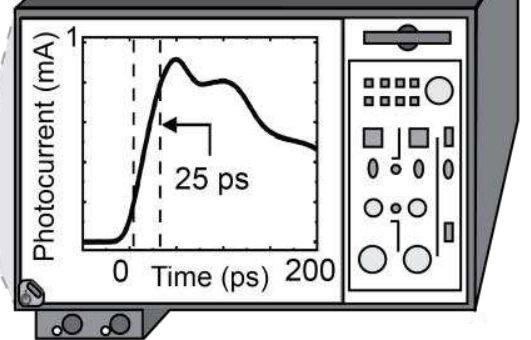

Fig. 1: A schematic of the ultrafast photocurrent spectroscopy (UPCS) to characterize carrier drift dynamics. (a) The ultrafast optical spectroscopies including photoluminescence and pump-probe mechanisms, enable characterization of short-range carrier diffusion dynamics. (b) UPCS enables in-situ characterization of the long-range carrier drift dynamics. The electron as a carrier is schematically depicted as an example in a lattice structure. (c) A lattice structure and transmission electron microscopy image of a $\mathrm{CsPbI}_{3}$ nanocrystal array (scale bar $\sim 10 \mathrm{~nm}$ ). (d) The UPCS with sub-25 ps time resolution is comprised of a high-speed co-planar waveguide, gold $(\mathrm{Au})$ electrodes, and a nanocrystal thin film that is illuminated by an ultrafast laser pulse to a generate photocurrent that is collected by a sampling oscilloscope. The laser beam covers the entire device including the nanocrystal film and the Au electrodes. 
In this work, we uses ultrafast photocurrent spectroscopy (UPCS) with a sub-25 ps time resolution to unravel the ultrafast carrier drift dynamics in perovskite nanocrystal photoconductive devices to address whether the underlying mechanisms present in solution processed $\mathrm{CsPbI}_{3}$ perovskite nanocrystal films are similar to those present in classic crystalline semiconductors. We aim to bridge two gaps in knowledge: 1) The similarity between solution-processed perovskite and classic crystalline semiconductors with respect to their transport properties, and 2) the relationship between ultrafast carrier dynamics and in operando devices. We use inorganic $\mathrm{CsPbI}_{3}$ nanocrystal arrays as a model system (Lattice structure and SEM image are shown in Fig. 1c). Unlike bulk perovskites, they have a wider tunability in terms of nanocrystal size, structure (quantum dots, layered materials, nanotube), surface chemistry, in addition to composition tunability. We discover that before defect states trap carriers, the $\mathrm{CsPbI}_{3}$ nanocrystal thin films exhibit similar carrier transport properties as $\mathrm{GaAs}$ and $\mathrm{Si}$. In the ultrafast temporal region, carriers undergo band-like transport due to the interaction of carriers with longitudinal optical (LO) phonons that dominate the higher temperature region $(>240 \mathrm{~K})$. In the lower temperature region $(>240 \mathrm{~K})$, carrier 'hopping' due to defect scattering dominates transport. These regions are delineated by a transition temperature that is unique to $\mathrm{CsPbI}_{3}$. Although all three semiconductors exhibit carrier-LO phonon interactions, $\mathrm{CsPbI}_{3}$ nanocrystal thin films demonstrate the strongest interaction compared to among these semicondcutors. In $\mathrm{CsPbI}_{3}$ nanocrystals, carriers preserve band-like transport, leading to a drift length larger than $125 \mathrm{~nm}$ with mobility $>25 \mathrm{~cm}^{2} / \mathrm{Vs}$.

\section{Results}

As shown in Fig. 1d, by integrating a $\mathrm{CsPbI}_{3}$ nanocrystal thin film photoconductor into transmission line architecture as a high-speed waveguide, the carrier drift dynamics can be investigated in photoconductors in operando with a sub-25 ps time resolution by the UPCS. The UPCS is superior to the Auston photoconductive switch technique since it has a much higher temporal resolution and can characterize various materials systems(21-23). A detailed nanocrystal film morphology and experimental configuration can be found in Supplementary Methods and Supplementary Fig. 1. Fig. 2a shows the various temperature-dependent ultrafast photocurrent curves for $\mathrm{CsPbI}_{3}$ nanocrystal film. Its typical photocurrent can be characterized by a fast rise to $\sim 25$ ps followed by a rapid decay up to $125 \mathrm{ps,} \mathrm{and} \mathrm{then} \mathrm{a} \mathrm{slow} \mathrm{tail} \mathrm{extending} \mathrm{beyond} 600 \mathrm{ps}$ (zero time is at $10 \%$ of the peak photocurrent). These three temporal regions are denoted by dashed vertical lines in Fig. 2a. In the first temporal region I, during the initial rise to $\sim 25$ ps, the peak photocurrent $\left(I_{\text {peak }}\right)$ decreases with increasing temperature from 240 to $400 \mathrm{~K}$, as shown in Fig. 2b, and can be described as

$$
I_{\text {peak }} \sim T^{-n}
$$

where $n$ is an index and $T$ is the temperature. This classic power-law relation is the signature of band-like transport due to carrier-phonon scattering. At the same time, the $n$ value indicates the strength of the carrier-phonon interaction, as discussed further below. Supplementary Fig. 2 depicts a complete photocurrent decay with an average carrier lifetime in the temperature range from $80 \mathrm{~K}$ to $400 \mathrm{~K}$ (see Supplementary Table 1). In region II, two competing transport mechanisms govern the photocurrent response between $\sim 25$ to $\sim 125 \mathrm{ps}$. On the one hand, the photocurrent dependence continues to decrease with increasing temperature, indicating the presence of phonon scattering. On the other hand, the time decay (Supplementary Fig. 3) shows an opposite trend; it increases with increasing temperature, which is a signature of hopping transport due to defect scattering. An Arrhenius relation for decay time with temperature can be written as

$$
\tau \sim e^{-\frac{\Delta E}{k_{B} T}}
$$


where $\Delta E$ is the activation energy, $k_{\mathrm{B}}$ is the Boltzmann constant, and $\tau$ is the decay time constant. The thermal activation energy of $\sim 1.8 \mathrm{meV}$ is derived in Supplementary Note 1 and Supplementary Fig. 3. In region III, i.e., beyond $\sim 125$ ps, the slow decay tail can be attributed to a hopping transport mechanism with an activation energy of $\sim 4.5 \mathrm{meV}$.

a

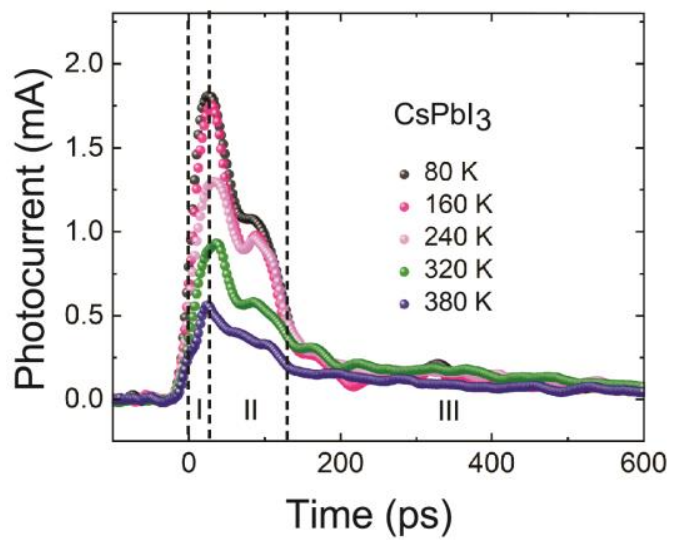

C

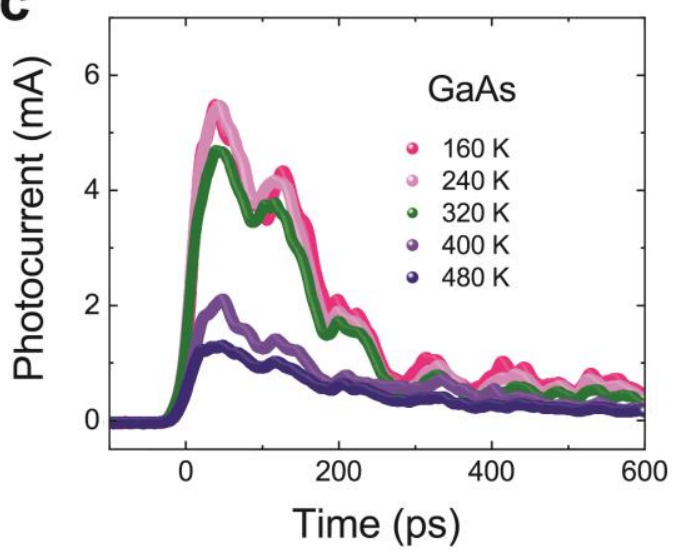

e

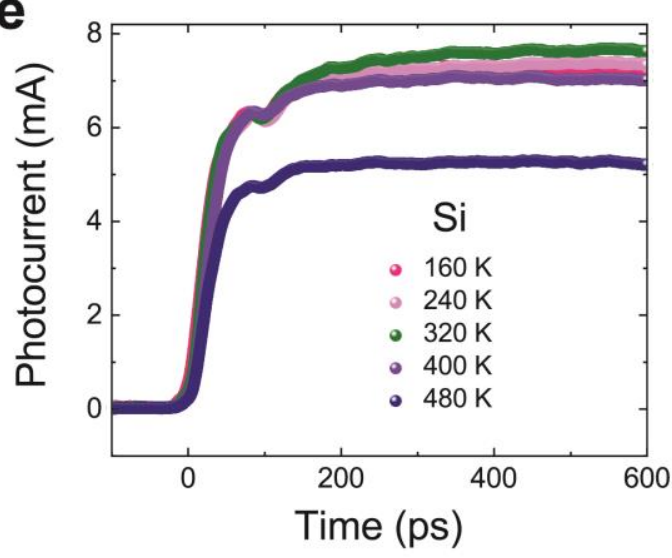

b
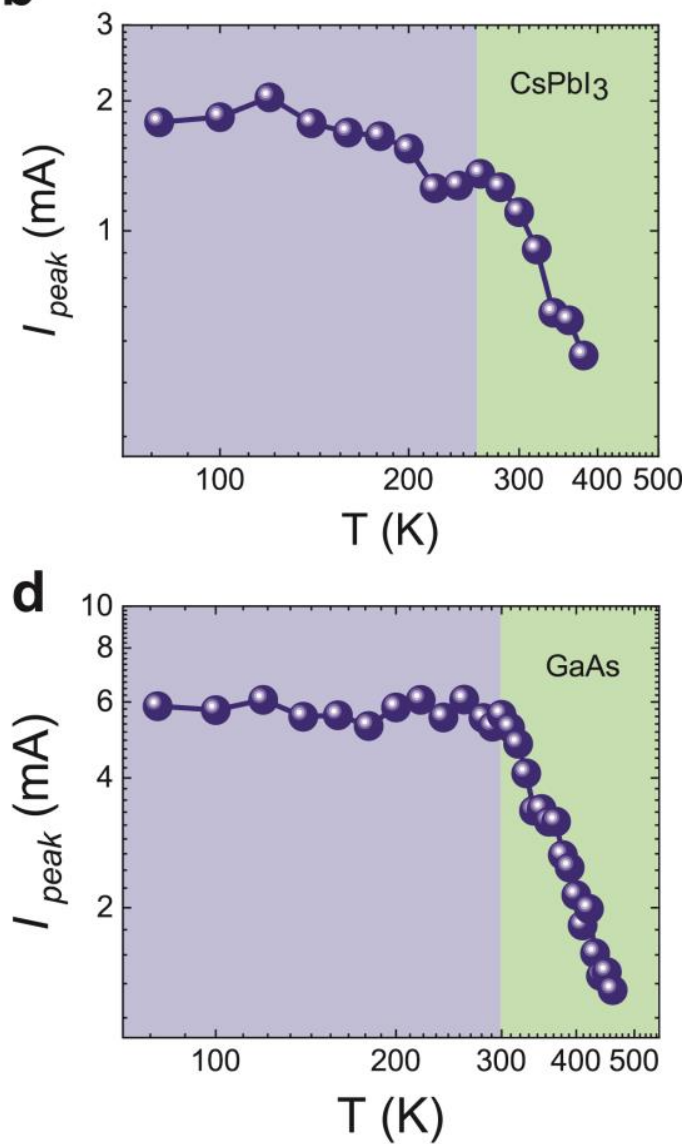

f

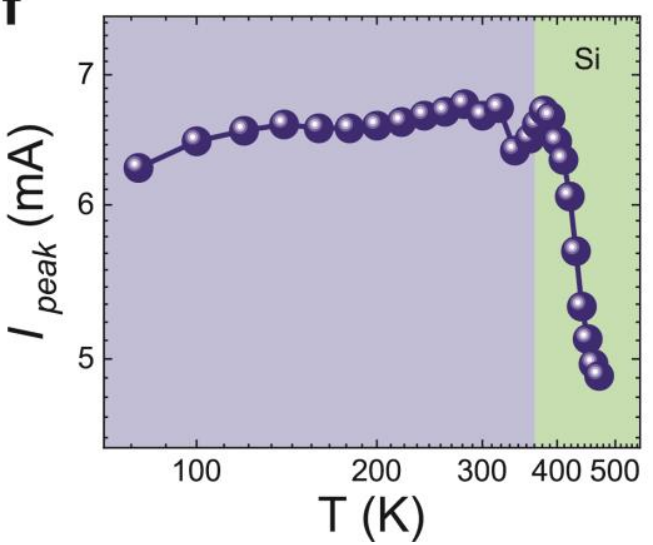

Fig. 2: Ultrafast photocurrent dependence on temperature. (a, c, e) Temperaturedependent ultrafast photocurrents for $\mathrm{CsPb}_{3}$ nanocrystal films, doped-GaAs, and lightly doped-Si, respectively. The corresponding temperature-dependent photocurrent peak on a $\log -\log$ scale is shown in panels $\mathrm{b}, \mathrm{d}$, and $\mathrm{f}$. 
To demonstrate the significance of events before trapping (based on the data we consider initial trapping occurs in region I as depicted in Fig. 2a), we compare the ultrafast photocurrents of $\mathrm{CsPbI}_{3}$ nanocrystal films with the traditional bulk semiconductors under the same electrical field and the photon density in the range of $10^{16} \mathrm{~cm}^{-3}$ (Figs. 2c and e). For doped-GaAs with a defect density $>10^{15} \mathrm{~cm}^{-3}$, the temperature-dependent photocurrent decay trend is similar to the $\mathrm{CsPbI}_{3}$ nanocrystal films, i.e., a fast rise followed by a rapid decay. Similarly, the photocurrent peak decreases with increasing temperature in the range from 300 to $500 \mathrm{~K}$ as illustrated in Fig. 2d. This can be attributed to carrier-phonon scattering. Moreover, the decay constant also increases as a result of defect scattering. In contrast, for slightly doped-Si with a defect density $<10^{15} \mathrm{~cm}^{-3}$, the photocurrent exhibits a fast rise, followed by a plateau up to a couple of ns, as shown in Fig. 2e. Like the $\mathrm{CsPbI}_{3}$ nanocrystal films and $\mathrm{GaAs}$, the photocurrent peak is attributed to phonon scattering since its amplitude decreases with increasing temperature (from $400 \mathrm{~K}$ to $500 \mathrm{~K}$ ), as illustrated in Fig. 2f. In addition to the temporal evolution of the photocurrent as seen in Figs. 2a, $\mathbf{2 c}$, and $\mathbf{2 e}$, the evolution of the peak photocurrent with temperature (Figs. 2b, 2d, and $2 \mathbf{f}$ ) is also an indication of the carrier transport mechanism. All three semiconductors exhibit a similar transition of the transport mechanism in the low temperature and high temperature regimes. For instance, the photocurrent peak of $\mathrm{CsPbI}_{3}$ nanocrystal films exhibits a weak temperature dependence below $240 \mathrm{~K}$. For doped-GaAs and slightly doped-Si, the transition temperature is 300 $\mathrm{K}$ and $380 \mathrm{~K}$, respectively. We attribute these transitions to a combination of defect scattering and acoustic phonon scattering at lower temperatures, which exhibit a temperature dependence opposite to that observed above the transition temperatures.

The mobility, which is a critical parameter to evaluate the carrier transport property, can be derived from the peak photocurrent as follows $(24)$ :

$$
I_{\text {peak }}=e \eta\left(\mu_{h}+\mu_{e}\right) \frac{N E}{L}
$$

where $\mu_{p}$ and $\mu_{e}$ are the hole and electron mobilities, respectively, $\mu=\left(\mu_{p}+\mu_{e}\right)$ is the total carrier mobility, $e$ is the electron charge, $\eta$ is the product of quantum efficiency of carrier photogeneration and carrier collection, $N$ is the effective photon number, $E$ is the electric field, and $L$ is the electrode spacing. For $\mathrm{CsPbI}_{3}$ nanocrystal thin films, conservatively calculated carrier mobility at room temperature is $\sim 2.5 \mathrm{~cm}^{2} / \mathrm{Vs}$. (assuming that the quantum efficiency is $100 \%$ so $\eta=1$ ) (See Supplementary note for calculations). Similarly, for GaAs and $\mathrm{Si}$, the calculated carrier mobilities at room temperature are $\sim 177 \mathrm{~cm}^{2} / \mathrm{Vs}$ and $\sim 264 \mathrm{~cm}^{2} / \mathrm{Vs}$, respectively. We note that these values are much lower than previously reported mobility values. For instance, the electron mobility is $3000 \mathrm{~cm}^{2} / \mathrm{Vs}$ for GaAs(25) and $\sim 1500 \mathrm{~cm}^{2} / \mathrm{Vs}$ for $\mathrm{Si}(26)$. The is due to the assumption that the quantum efficiency $(\mathrm{QE})$ of carrier photogeneration and collection is $100 \%$. For a $10 \% \mathrm{QE}$, the mobility for $\mathrm{CsPbI}_{3}$ nanocrystal thin films is $\sim 25 \mathrm{~cm}^{2} / \mathrm{Vs}$, which is in the same range as that characterized by the $\mathrm{THz} \operatorname{TDS}(11)$. Moreover, this high mobility value $\left(>10 \mathrm{~cm}^{2} / \mathrm{Vs}\right)$ at room temperature is consistent with the phonon scattering mechanism in $\mathrm{CsPbI}_{3}$ films, leading to bandlike transport. The linear dependence on electric-field and laser-intensity at $80 \mathrm{~K}$ for $\mathrm{CsPbI}_{3}$ nanocrystal thin films (Supplementary Figs. 5-8), as well the GaAs and Si (Supplementary Fig. 9), suggest that all three semiconductors exhibit the same photogeneration mechanism resulting from the free carrier generation or weak exciton with $<6.9 \mathrm{meV}(80 \mathrm{~K})$ binding energy, rather than an exciton model. 


\section{Carrier Phonon Interaction}

Since the hopping transport mechanism is well documented by the carrier trapping model in perovskite bulk films $(6,27,28)$ and nanocrystals $(29,30)$, here, we focus on pre-trapping dynamics to gain insights into the carrier-phonon interactions. The strength of the carrier-phonon interaction is related to the temperature-dependent carrier mobility $\left(\mu=\mu_{p}+\mu_{e}\right)$ as:

$$
\mu \sim T^{-n}
$$

As shown in Fig. 3, for $\mathrm{CsPb}_{3}$, in the temperature range of $240 \mathrm{~K}$ to $380 \mathrm{~K}, n=2.7$. At low temperatures $(<240 \mathrm{~K}), n=0.1$, which is attributed to a combination of defect scattering and acoustic phonon scattering (Supplementary Fig. 10). However, a striking feature is that the $n$ value of $\mathrm{CsPbI}_{3}$ is the highest compared to that of 1) traditional semiconductors, viz., $n=1.77$ and $n=$ 2.56 for $\mathrm{Si}$ and $\mathrm{GaAs}$, respectively; and 2) low-temperature solution-processed emerging perovskites such as $\mathrm{MAPbI}_{3}(n=1.53)(31)$ and $\mathrm{FAPbI}_{3}(n=1.64)(32)$. Apart from the above experimentally obtained $n$ values, first principle calculations starting from the Hamiltonian describing the relevant electronic bands and their interaction with phonons, computed using density functional theory (DFT) on $\mathrm{CsPbBr}_{3}$ by Motta and $\mathrm{Sanvito}(33)$ shows $n=1.47$. Moreover, some of the above have an $n$ index value much greater than 1.5, which is the benchmark for acoustic phonon scattering. Therefore, they all share a similar optical phonon-carrier scattering mechanism. Although the origin of this carrier-phonon scattering mechanism is still under debate, microscopic insight and quantitative analysis from first-principles calculations are still missing. Below we discuss reasons for the varying $n$ values and their relationship to the carrier-phonon scattering process.

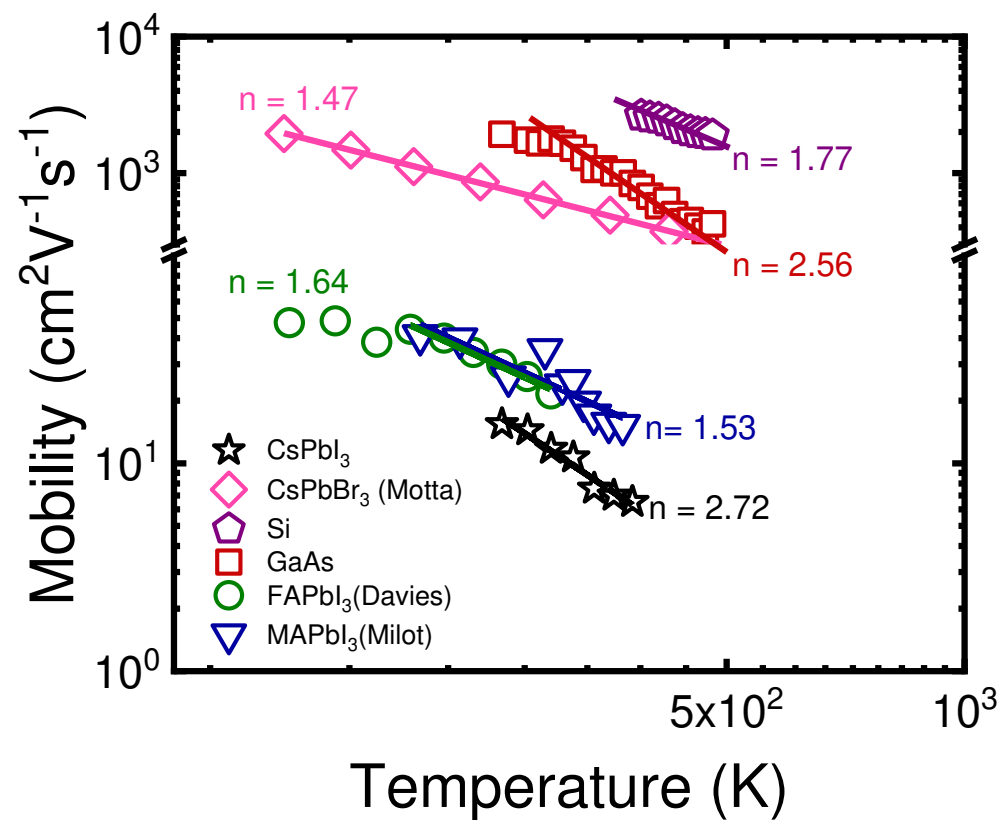

Fig. 3: Carrier mobility dependence on temperature. Carrier mobility dependence on temperature in two types of semiconductors: a typical crystalline polar semiconductor such as $\mathrm{GaAs}$, and $\mathrm{Si}$; theoretical $\mathrm{CsPbrr}_{3}$ (Motta); and solution processed $\mathrm{MAPbI}_{3}$ (Milot), $\mathrm{FAPbI}_{3}$ (Davies), and $\mathrm{CsPbI}_{3}$ nanocrystal perovskites.

In solution-processed emerging perovskites, the carrier-phonon coupling is due to the bond characteristics of the halide anion (in this case $\left.\mathrm{I}^{-}\right)$and the central organic $\left(\mathrm{MA}^{+}, \mathrm{FA}^{+}\right)$or inorganic 
$\left(\mathrm{Cs}^{+}\right)$cation. Two specific parameters determine the strength of the phonon scattering. They are i) the carrier-phonon coupling constant determined by the bond length $d$ of the cation(A)-halogen(X) bond (MA-I, FA-I, Cs-I) and the coordination number $a$ defining the number of bonds that connect $\mathrm{X}$ to $\mathrm{A}$; and ii) symmetric nature of the lattice. $\mathrm{In} \mathrm{CsPbI}_{3}, \mathrm{~A}$ is $\mathrm{Cs}^{+}$, which has a smaller radius but heavier ion (with respect to $\mathrm{MA}^{+}$), and its bonding with $\mathrm{I}-(\mathrm{X})$ preserves $\mathrm{PbI}_{6}{ }^{4-}$ symmetry as depicted in Supplementary Fig. 11 and Supplementary note 3. The large Cs-I bond length $(d \sim 4.5$ $\AA$ ) and weak dodeca-coordination with $\mathrm{I}^{-}$lead to a strong carrier phonon coupling. In comparison, $\mathrm{MAPbI}_{3}$, has the much lighter organic $\mathrm{MA}^{+}$cation, which interacts with $\mathrm{I}^{-}$by forming linear $\mathrm{I}-\ldots \mathrm{H} 3 \mathrm{~N}$, halide- hydrogen bonding perpendicular to the $\mathrm{Pb}^{2+}-\mathrm{I}^{-}-\mathrm{Pb}^{2+}$ cubic frame. This anisotropic perpendicular hydrogen bonding breaks the symmetry of the $\mathrm{PbI}_{6}{ }^{4-}$ lattice. In contrast, the smaller bonding length $(d \sim 2.75 \AA)$ and tri-coordination effectively suppress lattice vibrations leading to weaker carrier phonon coupling. Hence, when the organic cation $\mathrm{MA}^{+}$is replaced by $\mathrm{Cs}^{+}$an increased carrier-phonon scattering (increased $n$ index) is observed due to the enhanced $\mathrm{PbI}_{3}-\mathrm{LO}$ phonon mode coupling resulting from $\mathrm{Cs}^{+}-\mathrm{I}$ bonding and the strong vibration of the symmetric $\mathrm{PbI}_{6}{ }^{4-}$ lattice $(\mathrm{Pb}-\mathrm{I}$ cage) .

Unlike the perovskites, both GaAs and Si have face-centered cubic structures (zinc blend for GaAs and diamond for $\mathrm{Si}$ ) with bond lengths of $\sim 2.5 \AA$ and $\sim 3.8 \AA$, respectively. They are tetracoordinated and have a free vibrational structure (ions not confined to a cage). The symmetric nature of the bonding and strong ionic repulsion of $\mathrm{Ga}-\mathrm{Ga}$ and $\mathrm{Si}-\mathrm{Si}$ enhances LO lattice vibrations, resulting in a stronger carrier-phonon coupling than that of the anti-symmetric organic metal halide perovskites. Larger $n$ values indicate this coupling, but it is weaker (due to lower $d$ and $a$ ) when compared with $\mathrm{PbI}_{3}-\mathrm{LO}$ lattice vibrations in the symmetric $\mathrm{CsPbI}_{3}$. Hence, the $n$ values are smaller than that of $\mathrm{CsPbI}_{3}$. Additionally, the relationship between the $n$ value and the LO phonon mode is defined by Fivaz and Mooser(34) as,

$$
n_{\text {polar }}=\frac{\left(\frac{\hbar \omega_{\text {polar }}}{k_{B} T}\right) e^{\frac{\hbar \omega_{\text {polar }}}{k_{B} T}}}{\left(e^{\frac{\hbar \omega_{\text {polar }}}{k_{B} T}}-1\right)}-1
$$

where $\hbar$ is the reduced Planck's constant and $\omega_{\text {polar }}$ is the polar optical frequency. The LO phonon energy $\left(E_{\text {polar }}=\hbar \omega_{\text {polar }}\right)$ obtained from the above equation is $E_{\text {polar }} \sim 90 \mathrm{meV}$ (Supplementary note 3 and Supplementary Fig. 12). Although this value is larger than the $60 \mathrm{meV}$ as determined by temperature-dependent steady-state photoluminescence (Supplementary Fig. S13), the discrepancy may attribute to the nonequilibrium ultrafast timescale. Similarly, the derived LO phonon energy is $\sim 66 \mathrm{meV}$ for $\mathrm{Si}$, and $\sim 85 \mathrm{meV}$ for GaAs, respectively, and these values are consistent with the previous reports $(35,36)$.

\section{Carrier Transport Mechanism}

In Fig. 4, we identify the subtle ultrafast evolution of carrier drift length along an array of nanocrystals under an electrical field. We compare our results to the carrier diffusion length reported previously using ultrafast optical spectroscopies(37). The photogenerated carriers with excess energy typically thermalize to the band edge on a sub-ps timescale upon ultrafast laser excitation. At room temperature, from the sub-ps to sub- $25 \mathrm{ps}$ region, the calculated minimum drift length of the carrier under a moderate electrical field of $2 \times 10^{4} \mathrm{~V} / \mathrm{cm}$ with minimum mobility of $\sim 25 \mathrm{~cm}^{2} / \mathrm{Vs}$ is $\sim 120 \mathrm{~nm}$, which is equivalent to around ten nanocrystal width (each nanocrystal length is $\sim 12 \mathrm{~nm}$ and the separation is $\sim 0.6 \mathrm{~nm}$ as shown in Fig. 2c). Assuming that the band-like transport indicated by the temperature-dependent carrier mobility power law relation $\left(T^{-\mathrm{n}}, n=2.7\right)$ 
is maintained evenly throughout the entire drift length, the LO-carrier scattering period within each nanocrystal is $\sim 2.4 \mathrm{ps}$. The inter-nanocrystal carrier transport is tunneling transport mechanism, indicated by $n=0$, and the tunneling time is $\sim 0.1 \mathrm{ps}$. For reference, Zhang et al. reported a scattering time in $\mathrm{CsPbI}_{3}$ of $\sim 0.02 \mathrm{ps}$, characterized by THz-TDS(11). As a result, more than 100 scattering events in one nanocrystal can be reduced by further increasing the electric field, leading to a longer drift length. The scattering time found above is comparable to LO-carrier scattering times reported for $\mathrm{GaAs}(38)$ and $\mathrm{Si}(39)$, which corresponds well to similarities in the transport dynamics between $\mathrm{CsPbI}_{3}$ nanocrystal films and $\mathrm{Si}, \mathrm{GaAs}$ that was discussed previously.

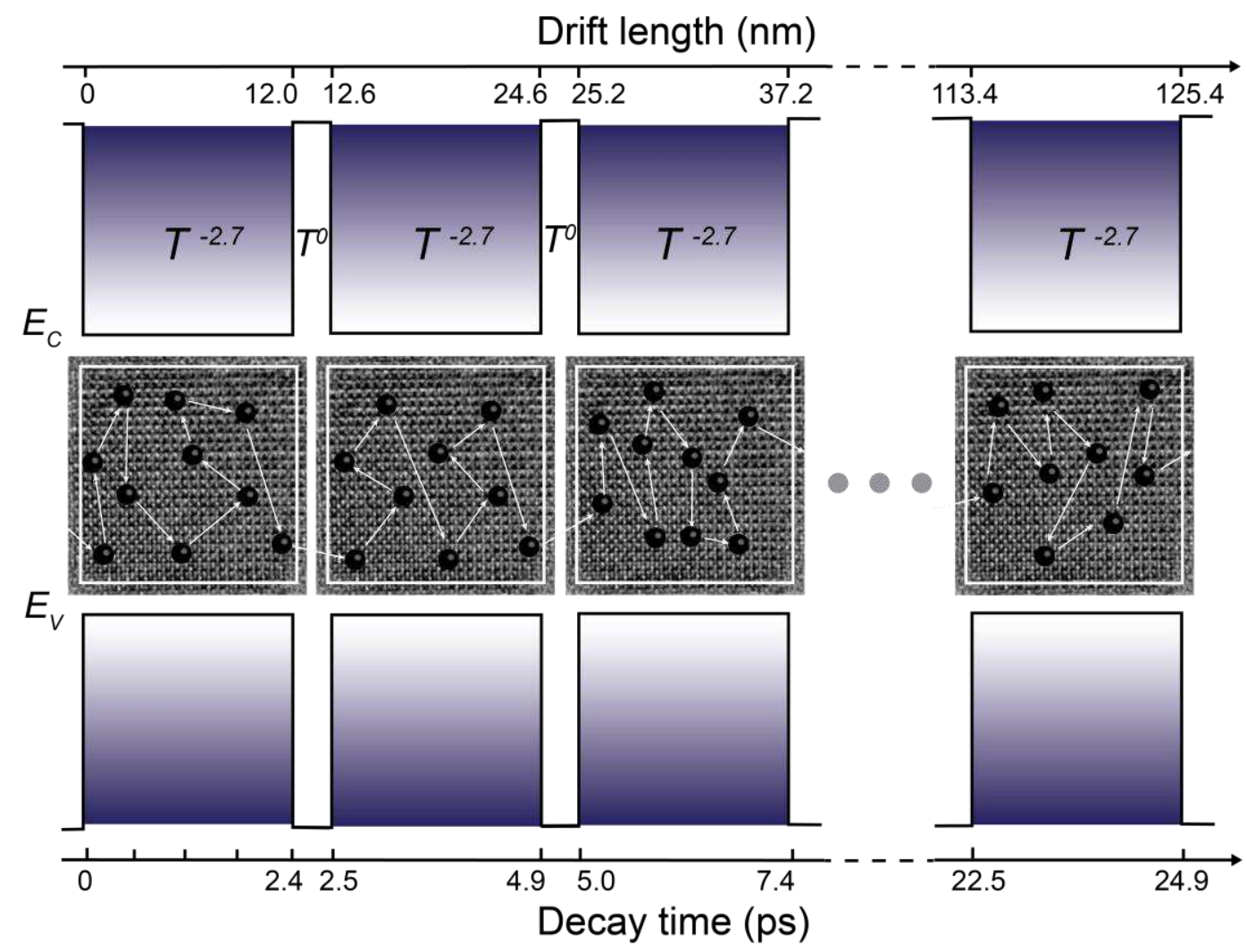

Fig. 4: The temporal and spatial evolution of the transport mechanism before carrier trapping. In the initial $\sim 25 \mathrm{ps}$, carrier transport is due to LO-carrier scattering mechanism within each nanocrystal, and tunneling among nanocrystals, resulting in a larger than $120 \mathrm{~nm}$ drift length. $E_{c}$ and $E_{v}$ are the conduction and valence band edges, respectively.

\section{Conclusion}

This ultrafast photophysics work has a profound implication for the photonic, optoelectronic, and electronic devices because perovskite devices such as solar cells, LEDs, X-ray, or photodetectors operate under the nonequilibrium conditions, where the carriers resides the excited state of band edges. By elucidating the nature of carrier drift dynamics and carrier-phonon interactions using the UPCS in operando photoconductive devices, our work establishes a foundation of the intrinsic electrical transport property in solution-processed perovskite and traditional semiconductors. The insights of intrinsic carrier transport mechanism will lead to a predictive discovery of emergent novel materials beyond perovskites through rational design of compositions, lattice structures, and 
atomic bonds. Our work also directly bridge the gap between carrier dynamics and the figure-ofmerit of devices including carrier mobility, lifetime, and drift length, etc. resulted from the in-situ characterization nature of the UPCS.

\section{Data and materials availability:}

All data are available in the main text or supplementary materials.

\section{Acknowledgments:}

K. K. P. A. and J. G. acknowledge the support from the South Carolina Research Authority (SCRA). We also wish to thank Russell Reynolds, Barrett Barker, and Michael Denz at Clemson University for their instrumental support. Q.Z. acknowledges fellowship support from the China Scholarship Council and Natural Science of Foundation China (21576140). M. C. B. and J. M. L. acknowledge the Center for Hybrid Organic Inorganic Semiconductors for Energy (CHOISE) an Energy Frontier Research Center funded by the Office of Basic Energy Sciences, Office of Science within the U.S. Department of Energy. Part of this work was authored by Alliance for Sustainable Energy, LLC, the manager, and operator of the National Renewable Energy Laboratory for the U.S. Department of Energy (DOE) under contract no. DE-AC36-08GO28308. The views expressed in the article do not necessarily represent the views of the DOE or the U.S. Government.

\section{Funding:}

South Carolina Research Authority (SCRA).

\section{Author contributions:}

K. K., E. L., and Q. Z. and performed the experiments. K. K., E. L., and Q. Z. contributed equally to this work. P. A, C. X., J. Z., Y. S., H. Z., D. L., H. S., M. H., T. C., O. C., R. R., A. M. R., M. C. B., and J. M. L. were involved in the technical discussion. J. G. supervised and designed the project and wrote the manuscript with K. K. All authors discussed the results and commented on the manuscript.

\section{Competing interests:}

The authors declare that they have no competing interests.

\section{References}

1. NREL, in NREL.gov. (NREL, 2020), vol. 2021.

2. Q. Chen et al., All-inorganic perovskite nanocrystal scintillators. Nature 561, 88-93 (2018).

3. G. Pacchioni, Highly efficient perovskite LEDs. Nature Reviews Materials 6, 108-108 (2021).

4. F. Wang, S. Bai, W. Tress, A. Hagfeldt, F. Gao, Defects engineering for highperformance perovskite solar cells. npj Flexible Electronics 2, 22 (2018).

5. J. M. Ball, A. Petrozza, Defects in perovskite-halides and their effects in solar cells. Nature Energy 1, 16149 (2016).

6. K. Kobbekaduwa et al., In-situ observation of trapped carriers in organic metal halide perovskite films with ultra-fast temporal and ultra-high energetic resolutions. Nature Communications 12, 1636 (2021). 
7. Y. Jia, R. A. Kerner, A. J. Grede, B. P. Rand, N. C. Giebink, Continuous-wave lasing in an organic-inorganic lead halide perovskite semiconductor. Nature Photonics 11, 784788 (2017).

8. Y. Yang et al., Observation of a hot-phonon bottleneck in lead-iodide perovskites. Nature Photonics 10, 53-59 (2016).

9. A. Kumar et al., Excitons in 2D perovskites for ultrafast terahertz photonic devices. Sci Adv 6, (2020).

10. Y. Lan et al., Ultrafast correlated charge and lattice motion in a hybrid metal halide perovskite. Sci Adv 5, (2019).

11. H. Zhang et al., Highly Mobile Large Polarons in Black Phase CsPbI3. Acs Energy Lett 6, 568-573 (2021).

12. T. A. Doherty et al., Performance-limiting nanoscale trap clusters at grain junctions in halide perovskites. Nature 580, 360-366 (2020).

13. C. B. Li et al., Insights into Ultrafast Carrier Dynamics in Perovskite Thin Films and Solar Cells. Acs Photonics 7, 1893-1907 (2020).

14. T. Morimoto et al., Microscopic ion migration in solid electrolytes revealed by terahertz time-domain spectroscopy. Nature Communications 10, 2662 (2019).

15. J. X. Shen, X. Zhang, S. Das, E. Kioupakis, C. G. Van de Walle, Unexpectedly Strong Auger Recombination in Halide Perovskites. Adv Energy Mater 8, (2018).

16. K. Miyata, T. L. Atallah, X. Y. Zhu, Lead halide perovskites: Crystal-liquid duality, phonon glass electron crystals, and large polaron formation. Sci Adv 3, (2017).

17. A. Musiienko et al., Deciphering the effect of traps on electronic charge transport properties of methylammonium lead tribromide perovskite. Sci Adv 6, (2020).

18. M. Petrovic, T. Ye, C. Vijila, S. Ramakrishna, Influence of Charge Transport and Defects on the Performance of Planar and Mesostructured Perovskite Solar Cells. Advanced Energy Materials 7, (2017).

19. T. M. Clarke, C. Lungenschmied, J. Peet, N. Drolet, A. J. Mozer, A comparison of five experimental techniques to measure charge carrier lifetime in polymer/fullerene solar cells. Advanced Energy Materials 5, 1401345 (2015).

20. M. C. Beard, G. M. Turner, D. J. Norris, C. A. Schmuttenmaer, Photoconductivity in CdSe nanoparticles studied by time-resolved terahertz spectroscopy (TRTS). Abstr Pap Am Chem S 219, U582-U582 (2000).

21. P. Adhikari et al., Sub-50 picosecond to microsecond carrier transport dynamics in pentacene thin films. Appl Phys Lett 113, (2018).

22. J. B. Gao, A. M. Rao, H. B. Li, J. B. Zhang, O. Chen, Carrier Transport Dynamics in High Speed Black Phosphorus Photodetectors. Acs Photonics 5, 1412-1417 (2018).

23. K. Kobbekaduwa et al., In-situ observation of trapped carriers in organic metal halide perovskite films with ultra-fast temporal and ultra-high energetic resolutions. Nat Commun 12, (2021).

24. D. Moses, Mechanism of carrier photogeneration in amorphous selenium: Fast transient photoconductivity. Physical Review B 53, 4462-4470 (1996).

25. A. Amith, I. Kudman, E. Steigmeier, Electron and phonon scattering in GaAs at high temperatures. Physical Review 138, A1270 (1965).

26. C. Jacoboni, C. Canali, G. Ottaviani, A. A. Quaranta, A review of some charge transport properties of silicon. Solid-State Electronics 20, 77-89 (1977).

27. H. D. Jin et al., It's a trap! On the nature of localised states and charge trapping in lead halide perovskites. Mater Horiz 7, 397-410 (2020). 
28. A. J. Knight, J. B. Patel, H. J. Snaith, M. B. Johnston, L. M. Herz, Trap States, Electric Fields, and Phase Segregation in Mixed-Halide Perovskite Photovoltaic Devices. Adv Energy Mater 10, (2020).

29. D. Giovanni et al., Origins of the long-range exciton diffusion in perovskite nanocrystal films: photon recycling vs exciton hopping. Light-Sci Appl 10, (2021).

30. G. C. Xing et al., Long-Range Balanced Electron- and Hole-Transport Lengths in Organic-Inorganic CH3NH3PbI3. Science 342, 344-347 (2013).

31. R. L. Milot, G. E. Eperon, H. J. Snaith, M. B. Johnston, L. M. Herz, Temperaturedependent charge-carrier dynamics in $\mathrm{CH} 3 \mathrm{NH} 3 \mathrm{PbI} 3$ perovskite thin films. Advanced Functional Materials 25, 6218-6227 (2015).

32. C. L. Davies et al., Impact of the organic cation on the optoelectronic properties of formamidinium lead triiodide. The journal of physical chemistry letters 9, 4502-4511 (2018).

33. C. Motta, S. Sanvito, Electron-phonon coupling and polaron mobility in hybrid perovskites from first principles. The Journal of Physical Chemistry C 122, 1361-1366 (2018).

34. R. Fivaz, E. Mooser, Mobility of charge carriers in semiconducting layer structures. Physical Review 163, 743 (1967).

35. D. N. Mirlin, I. J. Karlik, L. P. Nikitin, I. I. Reshina, V. F. Sapega, Hot electron photoluminescence in GaAs crystals. Solid State Communications 37, 757-760 (1981).

36. J. M. Hinckley, J. Singh, Monte Carlo studies of ohmic hole mobility in silicon and germanium: Examination of the optical phonon deformation potential. Journal of applied physics 76, 4192-4200 (1994).

37. B. Li et al., Surface passivation engineering strategy to fully-inorganic cubic CsPbI3 perovskites for high-performance solar cells. Nature Communications 9, 1076 (2018).

38. J. Sjakste, N. Vast, V. Tyuterev, Ab initio method for calculating electron-phonon scattering times in semiconductors: Application to $\mathrm{GaAs}$ and $\mathrm{GaP}$. Physical review letters 99, 236405 (2007).

39. A. S. Henry, G. Chen, Spectral phonon transport properties of silicon based on molecular dynamics simulations and lattice dynamics. Journal of Computational and Theoretical Nanoscience 5, 141-152 (2008). 


\section{Supplementary Files}

This is a list of supplementary files associated with this preprint. Click to download.

- SINatureNano.pdf 